\title{
The Role of Alcohol in Hippocampal Calcium Channel (Cav1.2) expression
}

\author{
Ryan Kokoska ${ }^{1}$, Eric Rodriguez ${ }^{2}$, Bryan Yamamoto ${ }^{2}$ \\ ${ }^{1}$ Indiana University School of Medicine; ${ }^{2}$ Indiana University School of Medicine, Department of \\ Pharmacology and Toxicology
}

\section{Background and Hypothesis:}

Voltage-gated L-type calcium channels $\left(\mathrm{Ca}_{v} 1.2\right.$ and $\left.\mathrm{Ca}_{v} 1.3\right)$ in the hippocampus play important roles in glutamatergic neurotransmission underlying memory and learning. Their overexpression has been implicated in neuroexcitatory cell death and disease states including chronic alcoholism. While increases in $\mathrm{Ca}_{\mathrm{v}} 1.2$ gene expression have been reported in the hippocampus after chronic ethanol exposure in rats, the regional distribution of $\mathrm{Ca}_{\mathrm{v}} 1.2$ protein after voluntary ethanol $(\mathrm{EtOH})$ drinking has not been reported. We hypothesize that the expression of $\mathrm{Ca}_{v} 1.2$ channels within the hippocampus is increased by EtOH drinking in a region-specific manner.

\section{Methods:}

Male Sprague Dawley rats were allowed 28 days of intermittent access to a $10 \% \mathrm{EtOH}$ solution. At 24 hours after the last exposure to $\mathrm{EtOH}$, brains were collected and processed for immunohistochemistry. Ca 1.2 associated immunofluorescent signal from subregions of the hippocampus was quantified using ImageJ analysis software.

\section{Results:}

Immunohistochemical results indicate that $\mathrm{Ca}_{v} 1.2$ immunoreactivity in the hippocampal stratum granulosum layer within the Dentate Gyrus and the stratum pyramidale layer within CA1 and $\mathrm{CA} 3$ regions was increased in response to $\mathrm{EtOH}$ treatment. There was no significant change in $\mathrm{Ca}_{\mathrm{v}} 1.2$ immunoreactivity for the CA2 region.

\section{Conclusion:}

This study suggests that calcium signaling in subregions of the hippocampus is differentially affected by $\mathrm{EtOH}$ consumption that may contribute to eventual calcium-mediated apoptosis.

\section{Impact and Implications:}

Understanding the process of $\mathrm{EtOH}$-induced hippocampal calcium signaling presents opportunities for understanding the consequences of chronic alcohol exposure related to hippocampal function including memory and learning, and possible interventional therapies for alcohol damage. 